\title{
TRANSFORMING BIG DATA INTO KNOWLEDGE USING SEMANTIC STREAM PROCESSING TECHNOLOGY: CHALLENGES AND EARLY PROGRESS
}

\author{
Paula-Georgiana ZĂLHAN \\ Babeș-Bolyai University of Cluj-Napoca, Romania \\ paula.zalhan@econ.ubbcluj.ro
}

\begin{abstract}
In the Big Data era, the volume of streaming data generated from heterogeneous data sources such as social media, smart devices or sensor networks is overwhelming but enables human users and applications to better understand the world around us. Understanding the context, situation or the environment where the data has been produced is a challenging task due the dynamic and fast nature of the streaming data. Modelling the big data using RDF model and applying continuous SPARQL queries over it using Semantic Web tools helps create machine-understandable meanings and valuable insights of the underlying data. This paper provides an overview of the Big Data theoretical foundation, challenges and stages that need to be taken into consideration when building a Big Data pipeline architecture. Also, the research efforts that have been made in the past years using Semantic Stream Processing techniques are described.
\end{abstract}

Keywords: Big data, RDF, Semantic stream processing, Semantic Web, SPARQL JEL classification: $\mathrm{C} 55, \mathrm{C} 88$

DOI: $10.12948 / \mathrm{ie} 2019.06 .04$

\section{Introduction}

Increasingly, applications require real-time processing of streaming data coming from multiple sources such as social media feeds (e.g. Twitter feed), cloud source repositories, public web, sensor networks, and connected Internet of Things (IoT) devices. These streaming sources generate big data volumes that need to be queried continuously for quickly detection of critical patterns or changing environmental conditions. For example, in Smart Healthcare domain, sensors are sending data to remote patient monitoring systems to observe patients' health condition in real-time. Also, click events generated by a website's visitors need to be processed in a timely fashion to improve the user experience by making real-time recommendations.

In response to the need of handling dynamic and infinite data with low processing-latency, the stream processing paradigm has emerged. Stream processing, also known as real-time streaming analytics, is a Big Data processing paradigm that deals with processing of large volumes of data within a time-constraint using distributed resources. This paradigm adopts the one-a-time processing model which applies continuous queries to the incoming data in order to get immediate results upon their arrival [27].

However, the dynamic nature of streaming data gathered from diverse sources makes the harvesting of the data a challenging task. In the last decade, the Semantic Web community has channelled its efforts to lift the streaming data generated by interconnected devices (e.g., sensors) to a semantic level using domain specific knowledge in order to enable applications, machines and human users to better understand the environment where the data was generated. Understanding the data context, potentially enables applications to take real-time decisions (e.g., to take rapid actions if critical patterns are detected). In order to remediate de problem of 
too much streaming data and not enough knowledge about its underlying meaning, the Semantic Stream Processing (SSP) research trend has emerged. Semantic stream processing refers to applying a set of models, principles and processing techniques to provide a machineunderstandable representation of the raw stream data, together with a description of the object that generated that data, to share and integrate information, and to infer new knowledge related to the physical world where the streams were captured [11].

The rest of the paper is organized as follows: Section 2 highlights some theoretical aspects related to the concept of Big Data and discusses the challenges of processing big data-sets. Section 3 presents the Big Data pipeline architecture to process streaming data. Section 4 describes the progress and developments that have been made in the past few years in the field of semantic stream processing. Finally, Section 5 concludes the paper.

\section{Big Data: Theoretical background}

This section is devoted to some of the main concepts related to big data including a definition of the term and its essential characteristics.

Big Data is a general term which has been introduced due to massive amounts of complex data that conventional data processing tools and techniques are not able to store and process them efficiently [17]. In 2010, Apache Hadoop [4] community defined Big Data as "datasets that could not be captured, managed and processed by general computers within an acceptable scope". According to the National Institute of Standards and Technology (NIST), "Big data shall mean the data of which the data volume, acquisition speed, or data representation limits the capacity of using traditional methods to conduct effective analysis".

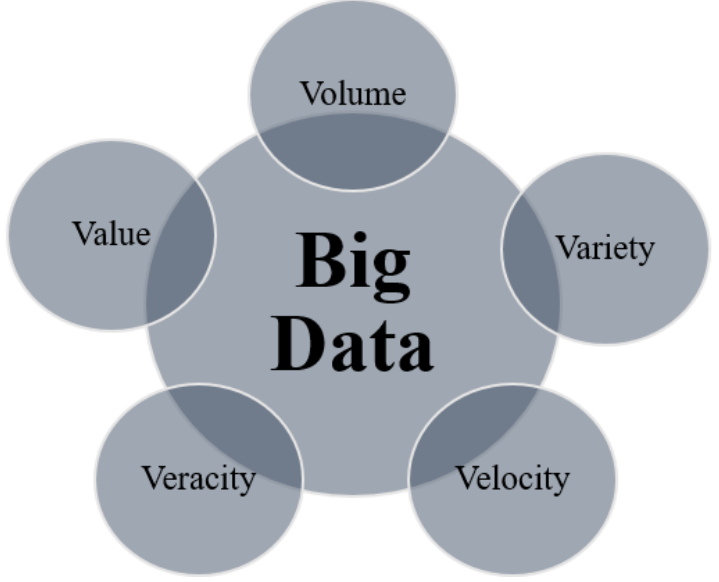

Figure 2. Essential characteristics of Big Data

Big data has complex features which make handling of it challenging. In recent years, industry experts and researchers have identified the big data's essential characteristics which can be summarized in the five V's as can be seen in Error! Reference source not found.: Volume, ariety, Velocity, Veracity and Value. Volume is related to the quantity or the size of the data which has to be processed. Big Data is related to enormous data sets, in the order of petabytes, zettabytes or above, which are impossible to store and handle using traditional data management systems [34]. Data variety refers to heterogeneous sources of data, both structured and unstructured, such as sensors, spreadsheets and databases, RFID tags, application logs, social media sites etc. Nowadays, data exist in different formats such as photos, videos, audio, text or other kinds of machine generated data which increase the complexity of the data analysis. Velocity essentially refers to the speed of the data generation. Data is generated with a fast rate, and continuously is ingested into a real-time processing system for querying and analysis. Veracity is concerned with the quality and the trustworthiness of data on which 
www.conferenceie.ase.ro

decisions are based [15]Error! Reference source not found. In other words, veracity uestions if the content of the big data is accurate enough to rely on when performing advanced data analytics. Last, but certainly not least is the value that Big Data offers. Systems must not only be designed to collect and process massive data efficiently but to make worth of the gathered data by extracting meaningful insights in real-time.

\section{Big Data pipeline to process streaming data}

Building an effective Big Data pipeline architecture requires to address several aspects including data generation, data processing and storage, and data visualization. The Big Data pipeline comprises several stages which are presented in Error! Reference source not found.. $\mathrm{n}$ the remainder of this section, each of these stages are briefly explained.

\subsection{Data ingestion stage}

In this stage, streaming data is collected from multiple data sources and imported into the system. Streaming data include a variety of data such as log files generated by customers using mobile or web applications, information from social media platforms, data gathered from sensor networks or other connected devices, geospatial services, etc. There are multiple tools that can be used as data ingestion systems such as Apache Kafka [5], Apache Nifi [6] and Apache Flume [3]. The popular data aggregation framework is Apache Kafka, publishsubscribe messaging platform that connects multiple message producers or data sources with multiple consumers or data sinks. The biggest benefit of Kafka is its ability to handle largescale data by providing read and write scalability. A close second is using replication to guard against data loss due to system failures [28].

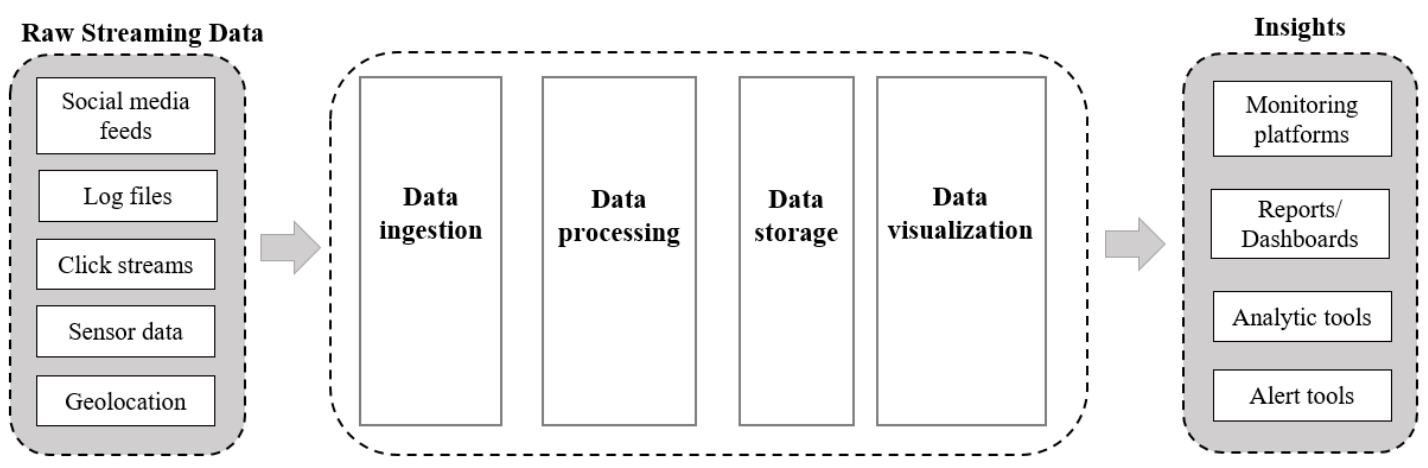

Figure 3. Big Data pipeline to process streaming data in real-time

\subsection{Data processing and data storage stages.}

In the data processing stage, streaming operations are applied to the incoming data including data filtering, transformations, aggregations, joins, and windowing. There are numerous distributed and parallel processing tools with the ability of stream processing over ingested data, performing sophisticated processing tasks on data and storing the intermediate results in memory, including Apache Spark [7], Apache Storm [8], and Apache Flink [2]. After processing the data, the real-time processing system persist the significant data streams into a distributed database. Depending on the processing that was executed on the streaming data, the storage can be a NoSQL database, such as Apache Cassandra [1], or a semantic database such as GraphDB [22].

\subsection{Data visualization stage}

In this stage the processed data is made available for consumption, where advanced analytics and monitoring platforms can be used to gain meaningful insights over the stored data. For 
example, human users may need to explore continuous real-time updates, or to see the output of the query results on a report/dashboard in order to take decisions timely. In this way, if critical patterns are discovered in the underlying data, alert tools should notify the consumer to take rapid actions.

\section{Semantics for Big Data}

In the past few years, a series of research efforts were focused on creating semantic technologies for processing the streaming data. The most consolidated group of research findings on Semantic Stream Processing include contributions in (1) continuous query models, languages and prototypes, using extensions of SPARQL [33] and collected under the RDF Stream Processing (RSP) label, and (2) stream reasoning techniques over data-streams.

\subsection{RDF Stream Processing}

A new research trend in the Semantic Web community, called RSP or Linked Data Stream Processing [26], was introduced to handle big data that can be modelled by means of the RDF model [31]. Multiple centralized RSP systems including C-SPARQL [10], CQELS [23], and SPARQL stream [14] have emerged which allow applying continuous queries over RDF streams using extensions of SPARQL. A unifying query model is proposed in [18] to formally define the semantics of a RSP system using a SPARQL-extended query language called RSP-QL. The incapacity of traditional RSP engines to process the large volume of data-stream in parallel, multiple distributed RDF systems were proposed to enable concurrent queries over the incoming data. For example, CQELS Cloud system [24] uses Apache Storm and Strider [30] uses Apache Spark to parallelize the continuous execution of queries over RDF data streams in the Cloud.

Meanwhile, several middleware solutions were proposed to transform streaming data into RDF streams using Semantic Web tools. For example, DataTurbine [21] engine is a streaming data middleware that follows the "publish-subscribe" model to deliver the data in near real-time from sensors to the Cloud for later analysis. Another middleware solution called Linked Stream Middleware [25] was introduced to transform the raw sensory data into RDF streams using W3C's Semantic Sensor Networks Incubator Group [32] ontology. Later, Ztreamy [20] system was developed by for large scale publishing of semanticallyannotated data streams on the Web.

\subsection{Semantic Reasoning}

Another research trend called Stream Reasoning [19] has emerged in the field of SSP in order to address the highly dynamic nature of data streams using inference techniques. For example, LARS framework was proposed by [12] as a formal foundation to express and analyse rich stream reasoning primitives using Answer Set Programming. Another system called Streaming MASSIF [13] was introduced to perform expressive reasoning and complex event processing over large amounts of heterogeneous IoT data using Cascade Reasoning approach.

A remarkable extension to logic-based reasoning is presented in [9] where authors use a combined approach of deductive and inductive stream reasoning to support analysis of streaming data. In order to infer new knowledge, logical deduction over streams implies reasoning from RDF statements while the inductive stream reasoning involves mining of large data sets and applying machine learning techniques. The author's approach has been applied to social media analysis, demonstrating the accuracy of the reasoner in this context. A similar effort is highlighted in [16] where authors focus on supervised stream learning from semantics of live traffic data using Description Logic (DL) reasoning in order to tackle the problem of concept drift. 
www.conferenceie.ase.ro

\section{Conclusions}

Performing streaming analytics over the data in motion must take into consideration the variety of data sources and the characteristics of Big Data. However, the heterogeneity of the data, as well as its vast and dynamic nature makes the problem of representation, integration and reasoning upon data a challenging task. To overcome these challenges, the streaming data is semantically enriched using Semantic Web tools stack, by providing machine-readable descriptions related to the context or environment where the data has been generated.

In the last decade, a lot of efforts from Semantic Web community were focused on creating tools and intelligent processing techniques to transform raw data into actionable knowledge. Many researchers have proposed centralized and distributed RSP engines to model the big data by means of the RDF model and execute continuous queries over the incoming data using SPARQL extensions. Some efforts were channelled to surpass the problems of the existing query languages by creating a unifying and comprehensive query model. Also, middleware solutions were introduced to transform streaming data into RDF streams. Multiple stream reasoners were built to infer new knowledge upon data.

\section{References}

[1] Apache Cassandra, https://cassandra.apache.org/, [April 1, 2019]

[2] Apache Flink, https://flink.apache.org/, [April 1, 2019]

[3] Apache Flume, https://flume.apache.org/, [April 1, 2019]

[4] Apache Hadoop, https://hadoop.apache.org/, [April 1, 2019]

[5] Apache Kafka, https://kafka.apache.org/, [April 1, 2019]

[6] Apache Nifi, https://nifi.apache.org/, [April 1, 2019]

[7] Apache Spark, https://spark.apache.org/, [April 1, 2019]

[8] Apache Storm, https://storm.apache.org/, [April 1, 2019]

[9] D. Barbieri, D. Braga, S. Ceri, E. Della Valle, Y. Huang, V. Tresp, A. Rettinger and H. Wermser, "Deductive and inductive stream reasoning for semantic social media analytics," IEEE Intelligent Systems vol. 25, no. 6, pp. 32-4, 2010.

[10] D.F. Barbieri, D. Braga, S. Ceri and M. Grossniklaus, "An execution environment for C-SPARQL queries," in Proc. The 13th International Conference on Extending Database Technology, 2010, pp. 441-452.

[11] P. Barnaghi, W. Wang, C. Henson and K. Taylor, "Semantics for the Internet of Things: early progress and back to the future," International Journal on Semantic Web and Information Systems, vol. 8, no. 1, pp.1-21, 2012.

[12] H. Beck, M. Dao-Tran, T. Eiter and M. Fink, "LARS: A logic-based framework for analysing reasoning over streams," in Proc. The 29th Conference on Artificial Intelligence, AAAI Press, 2015, pp. 1431-1438.

[13] P. Bonte, R. Tommasini, E. Della Valle, F. De Turck and F. Ongenae, "Streaming MASSIF: Cascading Reasoning for Efficient Processing of IoT Data Streams," Sensors, vol. 18, no. 11, pp. 3832, 2018.

[14] J.-P. Calbimonte, O. Corcho and A.J. Gray, "Enabling ontology-based access to streaming data sources," in Proc. The 9th International Semantic Web Conference, Springer, Berlin, Heidelberg, 2010, pp. 96-111.

[15] J. Cano. The V's of Big Data: Velocity, Volume, Value, Variety, and Veracity. Internet: https://www.xsnet.com/blog/bid/205405/the-v-s-of-big-data-velocity-volume-valuevariety -and-veracity/, March 11, 2014 [April 1, 2019] 
[16] J. Chen, F. Lécué, J. Pan and H. Chen, "Learning from ontology streams with semantic concept drift," in Proc. The 26th International Joint Conference on Artificial Intelligence, Sierra C, 2017, pp. 957-963.

[17] M. Chen, S. Mao and Y. Liu, "Big data: A survey," Mobile Networks and Applications, vol. 19, no. 2, pp.171-209, 2014.

[18] Dell'Aglio, D., Della Valle, E., Calbimonte, J.P. and Corcho, O.: RSP-QL semantics: A unifying query model to explain heterogeneity of RDF stream processing systems. Semantic Web and Information Systems. 10(4), 17-44 (2014)

[19] D. Dell'Aglio, E. Della Valle, F. van Harmelen and A. Bernstein, "Stream reasoning: A survey and outlook," Data Science, vol. 1, no.1-2, pp.59-83, 2017.

[20] J.A. Fisteus, N.F. García, L.S. Fernández and D. Fuentes-Lorenzo, "Ztreamy : A middleware for publishing semantic streams on the Web," Journal of Web Semantics: Science, Services and Agents on the World Wide Web, vol. 25, no. C, pp. 16-23, 2014.

[21] T. Fountain, S. Tilak, P. Shin and M. Nekrasov, "The open source dataturbine initiative: empowering the scientific community with streaming data middleware," The Bulletin of the Ecological Society of America, vol. 93, no. 3, pp. 242-252, 2012.

[22] GraphDB, http://graphdb.ontotext.com/, [April 1, 2019]

[23] D. Le-Phuoc, M. Dao-Tran, J.X. Parreira and M.Hauswirth, "A native and adaptive approach for unified processing of linked streams and linked data," in Proc. The 10th International Semantic Web Conference, Springer, Berlin, Heidelberg, 2011, pp. 370-388.

[24] D. Le-Phuoc, H. Nguyen Mau Quoc, C. Le Van and M. Hauswirth, "Elastic and Scalable Processing of Linked Stream Data in the Cloud," in Alani H. et al. (eds.) The Semantic Web-ISWC 2013, Lecture Notes in Computer Science, vol. 8218, Springer, Berlin, Heidelberg, 2013, pp. 280-297.

[25] D. Le-Phuoc, H.Q. Nguyen-Mau, J.X. Parreiraand and M. Hauswirth, "A middleware framework for scalable management of linked streams," Journal of Web Semantics: Science, Services and Agents on the World Wide Web, vol. 16, no. C, 2012, pp. 42-51.

[26] D. Le-Phuoc, J. Xavier Parreira and M. Hauswirth, "Linked Stream Data Processing," in Eiter, T., Krennwallner, T. (eds.) Reasoning Web. Semantic Technologies for Advanced, Query Answering. Reasoning Web 2012, Lecture Notes in Computer Science, vol. 7487, Springer, Berlin, Heidelberg, 2012, pp. 245-289.

[27] X. Liu, A.V. Dastjerdi and R. Buyya, "Stream processing in IoT: Foundations, stateof-the-art, and future directions", in Internet of Things: Principles and Paradigms. Cambridge: Morgan Kaufmann, 2016, pp. 145-161.

[28] N. Narkhede, G. Shapira and T. Palino, Kafka: The Definitive Guide: Real-time Data and Stream Processing at Scale. O'Reilly Media, Inc., 2017.

[29] S. Pacha, S. Ramalingam and R. Sethukarasi, "Semantic annotation of summarized sensor data stream for effective query processing," Journal of Supercomputing, 2017, pp. $1-23$.

[30] X. Ren and O. Cur, "Strider: A hybrid adaptive distributed RDF stream processing engine," in The International Semantic Web Conference, Springer, 2017, pp. 559-576.

[31] Resource Description Framework (RDF), https://www.w3.org/RDF/, [April 1, 2019]

[32] Semantic Sensor Network XG, http://www.w3.org/2005/Incubator/ssn/, [April 1, 2019]

[33] SPARQL 1.1 Query Language, https://www.w3.org/TR/sparql11-query/, [April 1, 2019]

[34] W. Vorhies. How many "V's" in Big Data? The Characteristics that define Big Data. Internet: http://www.datasciencecentral.com/profiles/blogs/how-many-v-s-in-big-datathe-characteristics-that-define-big-dat/, October 31, 2014 [April 1, 2019] 